\title{
Pervasive Nursing AND docToral Assistant - PINATA
}

\author{
Alexiei Dingli, Charlie Abela, Ilenia D’Ambrogio \\ Department of Intelligent Computer Systems, Faculty of ICT, \\ University of Malta \\ Msida, Malta \\ \{alexiei.dingli, charlie.abela, ilenia.dambrogio\}@um.edu.mt
}

\section{ABSTRACT}

The ratio of nurses and doctors to patients keeps diminishing due to increasing population health needs, however it is expected that the quality in healthcare services increases. By merging Ambient Intelligence (AmI) and semantic web technologies, PINATA aspires to address this issue. PINATA utilises pervasive devices to aid doctors and nurses to focus on the patient and thus improve the quality of healthcare services. In this paper we go over comparable AmI system architectures; summarise the physical and logical design of PINATA; provide details of the knowledgebase modelled in $\mathrm{RDF} / \mathrm{S}$ and the ontologies designed for units of interest, including resources, and context-related concepts. These individual models are unified into a connected, context-rich data model through another set of classes and properties. The results presented were based on several tests done and are very promising.

\section{KEYWORDS}

Ambient Intelligence, Health Care, Mobile Technology, Context Awareness, Semantic Web, Ontology

\section{INTRODUCTION}

The IST Advisory Group [1] claims that in an Ambient Intelligent (AmI) Environment, people will be surrounded by intelligent interfaces that are backed by networking technology and computing that is embedded in everyday items. They also maintain that an AmI environment is one that is responsive of the particular characteristics of human presence and personalities by altering according to the needs of the users. They say that such an environment must be able to react intelligently to signalled or verbal requests and maybe even engage in an intelligent dialogue while being unobtrusive. They also state that the interface should be comfortable and pleasant for the users without involving extreme learning curves.

Medical staff frequently share available resources and are very mobile. They regularly work collaboratively in teams. Since their work's focal point is the physical health of patients, digital content should offer help in decision processes [2]. Ambient intelligence is well suited to assist medical practitioners in a number of areas. Combined with mobile technologies, it can enhance patient identification processes, for instance by using RFID tags containing limited information about the patient and thus avoid mistreatments because of mistakes at some stage in treatment [3].

The Pervasive, Nursing and Doctoral Assistant (PINATA) is a system which uses AmI to aid doctors and nurses in upholding high levels of service [4]. This is achieved through a combination of Wi-Fi-enabled tags which are associated with, and worn by doctors, nurses and patients and Wi-Fi software tags, which are software clients installed on handhelds so as to achieve the same functionality as the physical tags. A particular kind of tag that reports location and temperature readings is utilised in the store's refrigerator to monitor its ambient. Passive RFID tags attached to pharmaceutical boxes are tracked by means of RFID handheld readers. The Kinect ${ }^{1}$ camera, which is a $3 \mathrm{D}$ camera, is used as an alternative to traditional video cameras, which produce a flat image. These are installed in patients' rooms, with the aim of monitoring and ensuring the patients' welfare. Handhelds are used by doctors and nurses to manage patients' records and to receive alerts when required. Keychain sized and shaped RFID readers are used in combination with these handhelds so as to provide interactivity with the various tagged items.

The rest of this paper is structured as follows: The relevant work section describes briefly various AmI related projects that are of interest to PINATA; the following section describes the infrastructure elements of PINATA, namely the different devices and network structures that are being utilised in this project; PINATA's architecture and knowledgebase are then detailed in the next section which is followed by the presentation of some preliminary evaluation.

\section{RELEVANT WORK}

In recent years, several research bodies have embarked on projects to investigate the benefits that AmI can bring about in diverse facets of life. Every project has its particular individual features and objectives. In this section, AmI projects will be considered, outlining their major contributions and relevance to PINATA.

Oxygen (2001-2004) was a research project intended to study technologies that facilitate pervasive and human-centric applications and was carried out by the Massachusetts Institute of Technology (MIT) in association with the

\footnotetext{
${ }^{1}$ http://www.xbox.com/en-US/kinect
} 
Laboratories for Computer Science (LCS) and that of Artificial Intelligence Laboratory (AIL). Oxygen's idea was to make computation and communication very accessible to humans by means of natural interfaces of vision and speech [5]. Thus computation is merged with peoples' lives allowing them to accomplish tasks like teamwork, retrieving knowledge, and automating their surroundings and routine everyday jobs easily [5]. Whereas the functioning objectives of Oxygen differ from those of PINATA, certain shared ideas between the two projects exist. Both projects utilise handhelds and while originally PINATA intended to use cameras like Oxygen, it was later decided to make use of Kinects instead since these are 3D cameras and offer better features than traditional ones. A bird's eye view of the building is used to help users find their way in an Oxygen scenario. This method is also used in PINATA to help members of staff find patients and objects.

Amigo (2004-2008) was a research project done by a conglomerate of fifteen European companies and research organisations in software development, mobile and home networking, consumer electronics and domestic appliances. [6]. It focused on the benefits of AmI in a home setting, with particular attention to scenarios involving information and entertainment, extendibility of the home setting and care and safety. This project's aims are nearer to PINATA's, in particular those related to safety and healthcare. Amigo features a context awareness module which uses transformation and context representation formalisms in conjunction with context ontologies. In a similar manner, PINATA models device context (e.g. handheld screen size), user context (e.g. classification of hospital users) and physical context (e.g. location of resources). Just like Amigo, PINATA also customises the output of information according to the current user and context.

Hydra (2006-2010) developed a middleware for networked embedded systems that can be used across several AmI applications [7]. The necessity for systems that are able to make environments more intelligent, while keeping userfriendly interfaces was the main motivation behind this project [7]. Various similarities between Hydra and PINATA can be noticed. Like in PINATA, a device ontology that stores metainformation about the software and hardware of the devices is an important component of Hydra. The ontologies are used to adapt the output according to the current context. Hydra's middleware was tested in three diverse fields, including that of healthcare, thus allowing for further comparison with PINATA.

The Connectivity Systems group of Philips Research Aachen, Germany are working on wireless networks of small sensors that monitor patients' vital signs, for example, cardiac activity [8]. Martin Elixmann, the head of the group, claims that these days, people are frequently hospitalised throughout their recovery exclusively because they require monitoring.
The author explains that because the sensors are wireless, the wearers can move about the hospital or even their home, liberally. He claims that current devices on the market allow mobility of patients; however they are only capable of recording data, whereby, no one will notice should the patient take a turn for the worse, in contrast to these sensor networks which are able to monitor patients in real-time and raise the alarm. One of PINATA's scenarios involving the admittance of a patient with an infectious disease could benefit from such sensor networks.

SALSA [9] is also a middleware, and it is designed to support AmI environments development based on autonomous agents. The team maintains that the healthcare professional faces a great level of mobility when working in hospitals, because of the need to access patients' records and resources which are dispersed all over the building, together with the need to continuously collaborate with colleagues. They put forward the idea that autonomous agents offer abilities of intelligence and proactivity to the healthcare environment which is supplied with ubiquitous computing and resources, giving rise to an AmI system, where the autonomous agents facilitate ubiquitous technology to react to users' specific circumstances and demands. PINATA also tackles the mobility of healthcare staff in a hospital setting and tries to facilitate access to patients' records and tracks the location of resources, patients and staff.

Honeywell Laboratories are developing a system where the software examines images of the daily activities of a person. If an unforeseen divergence from the pattern occurs, caregivers are warned [10]. This scenario is very similar to that tackled by the camera system, also referred to as the Visual Tracking System in PINATA

Hartog et al put forward another scenario, this time in the case of a medical emergency whereby AmI systems do not only store information but constantly monitor and assess a patient's health condition [11]. The proposed scenario foresees an ambulance being on the way to a scene in seconds and the updated reports, together with the medical history of the patient are exchanged between the ambulance staff, the hospital and the personnel concerned automatically and continuously. Through this idea, a more efficient service is provided because the healthcare professionals are capable of assessing and diagnosing patients before they even reach the hospital [12]. This scenario resembles the ones in PINATA where the sharing and updating of patients' medical history is synchronised between the central knowledgebase and the handhelds.

An intelligent Personal ECG Monitor (PEM), developed by the European EPI-MEDICS project, aims at early sensing of cardiac events [13]. Advanced decision-making techniques are embedded in PEM, through which it is possible to generate varied alarm levels and to send warning messages to the appropriate care providers via wireless communication 
[13]. The care provider is involved only if needed and the system requires no particular infrastructure, thus making PEM a cost-saving solution [13]. PEM is deemed a typical model of AmI and pervasive computing, indicative of how healthcare can be enhanced through ubiquitous devices which are personalised and wearable [13]. Besides sharing the same domain as PINATA, both projects use typical wireless communication to pass-on warning messages to caregivers. In a number of scenarios, PINATA monitors the location of patients and alerts staff whenever a patient enters in unauthorised areas of the hospital.

iHospital encapsulates the notion of a healthcare facility that helps the activity of hospital staff through information technology [14]. Continuous activity changing, the great quantity of information, the essential harmonization of activities with colleagues and high mobility are some of the features of a hospital setting outlined by the authors. The fundamental assumption here is that a hospital as a very interactive place of work, and through a variety of diverse devices, the staff can access medical information and work together with their colleagues taking into consideration contextual information. Relevant information is made available anytime and everywhere through an ubiquitous communication infrastructure. The authors deem the medical record as a main information element and therefore highlight the importance of facilities that provide access to such records and ways to update them. In addition, the location of people and artefacts and the tracking of the ongoing activities are monitored through the use of sensing devices so that pertinent contextual information can be derived, which can then be visualised on diverse devices [14]. iHospital has a lot of similarities to PINATA - the hospital setting, the derived features of the working environment, the diverse devices used, the use of contextual information and the need for access to medical records. iHospital makes use of devices such as large screens, ambient displays, and smartphones - which are however deemed as limited in screen size and performance [14]. The team behind PINATA opted for the latest internet tablets available on the market to be used as handhelds thus overcoming these restrictions.

AmICa (Ambient Intelligence platform for Cardiology) monitors cardiac patients [15]. It features a layered architecture consisting of the AmI server - the heart of the system that supplies the requisite functionality, the client and various devices [15]. This architecture is also similar to that of PINATA which is composed of the Knowledge Brokering Module that encompasses the Knowledgebase and Ambient Intelligence, the Device Manager and the devices used namely the RFID tags, handhelds and Kinects, as shown in Figure 1.

Table 6, in the Appendix summarises the features of the different works cited in this section in comparison to PINATA.

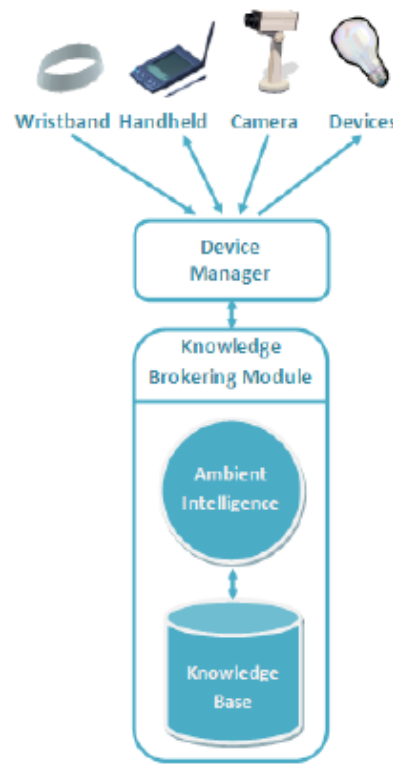

Figure 1 The PINATA architecture taken from [4]

V. INFRASTRUCTURE ELEMENTS

Context-awareness is of significant importance within AmI projects. Contextual information is acquired through hardware that is capable of sensing various contextual components, such as, light level, location and temperature. Furthermore, such systems call for unobtrusiveness. Hence, data transfers are done through wireless networks. This section examines PINATA's infrastructure detailing the devices and the types of networks utilised.

\section{A. DEVICES}

PINATA is composed of four device systems - two providing input and the other two provide both input and output. Hospital resources are tracked by the Real-Time Locating System (RTLS), while the Visual Tracking System (VTS) keeps an eye on the patients making sure of their welfare. Timely and context-aware information is presented to staff through handheld devices thus assisting them in their job. Guest devices brought into PINATA's range are also looked for and utilised.

\section{1) Real-Time Locating System (RTLS)}

Assets which have tags attached to them can be tracked and located via the RTLS. The movement of humans and objects inside the hospital is tracked within PINATA using such a system. Four major technologies used in locating systems were appraised: passive RFID, active RFID/Infra Red, Wi-Fi and Ultrasound. While all of the solutions examined could have been used for a number of PINATA's scenarios, none of them completely fulfilled the functional requirements. 
Though passive RFID tags proved appropriate to tag all assets including small consumables, all the other technologies outperformed the RFID technology when it came to proximity detection. Because of this shortcoming, we chose to adopt a hybrid solution where different resources are located using different technologies. Pharmaceuticals, for example, are being tracked using passive RFID tags while Wi-Fi was deemed the most suitable for tracking the rest of the resources, including patients and staff members.

\section{2) Visual Tracking System}

In recent years, computer vision has advanced with giant strides both in hardware and software. The constant improvement in cameras' picture quality means that today inexpensive webcams can be used for varied scenarios. The addition of a visual tracking component in PINATA was believed as essential owing to this technology's non-invasive qualities which makes it favourable to pure AmI solutions. At first, we had considered the use of wireless, IP cameras that exhibited night-vision, two-way audio, together with pan and tilt. However, the recent release of Kinects allowed for further consideration. The Kinect controller was preferred because of its reasonably priced sensing abilities. Through its utilisation, PINATA provides for the creation of a 3D map of the patient's room, the identification of the human presence in the room and the ability to respond to human movement or the lack of it.

\section{3) Handheld Devices}

Handheld computers are developing significantly, and recent developments allow them to handle much of the conventional desktop functionality. Some of these computers have as features extra input peripherals which could be linked to AmI systems, for example, RFID readers which are essential for location awareness, microphones which provide for sound awareness, and cameras which provide visual awareness. In PINATA, handhelds provide for portable and inconspicuous information access. Within PINATA handhelds are the most important output devices. They present an integrated view of all the information collected through the RTLS and camera modules. Since handhelds support Wi-Fi, they can assimilate with the Wi-Fi based RTLS system with no extra hardware tags required. However, since these handhelds were going to be used by doctors and nurses while on the go, a larger screen size was preferred. This immediately ruled out handheld devices which are equipped with RFID reading because they tend to have small displays. It was thus decided to go for internet tablets, so that it would be comfortable enough to work with and not too bulky to carry around. Since such handhelds do not come equipped with internal RFID capabilities, they require an external RFID reader. There are diverse readers currently on the market. We opted for a keychain sized and shaped reader that communicates with the tablets via Bluetooth.

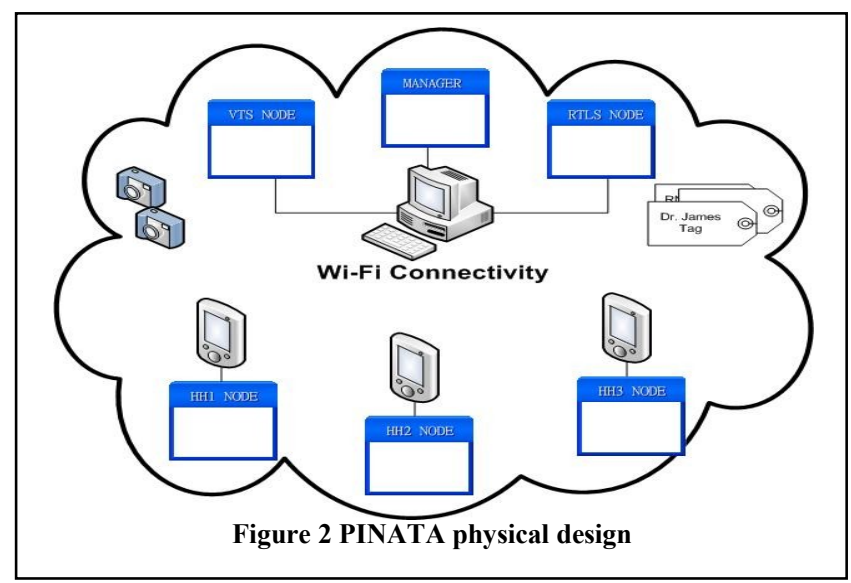

4) Guest Devices

PINATA is implemented to take advantage from guest devices, which can be considered as free resources, and through which it is possible to increase the general intelligence of the hospital environment and to offer novel services to visitors. Since mobile phones operational at present have a lot of diverse capabilities, network support and platforms, PINATA will query each device and alter its services according to the particular device.

\section{B. NETWORKS}

PINATA is mainly enabled to use the Wi-Fi network infrastructure for data communication between its diverse devices. Additionally, Bluetooth is used for communication with guest devices that do not support Wi-Fi, even though nowadays smart-phones, which are Wi-Fi-enabled, are on the increase.

\section{ARCHITECTURE}

The PINATA architecture is composed of various decentralised applications which are referred to as nodes and a centralised application referred to as manager. Nodes are custom-applications written purposely for a specific device or device system. As shown in Figure 2, the RTLS, the VTS, and each of the handheld and guest devices are considered as nodes. A desktop computer will host the nodes for the RTLS and VTS since they are both limited resources. In contrast, each handheld device hosts its own node since they are regarded as autonomous devices. The input nodes (RTLS, VTS and handhelds) will provide the required context while the handhelds act also as output nodes since they are reporting devices. The role of the manager is twofold: (i) to support the development of the nodes through the provision of shared libraries via web services and (ii) to coordinate the events and services of all the nodes. All communication within the system is through Wi-Fi. Consequently, Wi-Fi communication channels exist between the Wi-Fi tags and the desktop hosting the locating node; the VTS and the desktop hosting its node; and the desktop and each of the handhelds. 


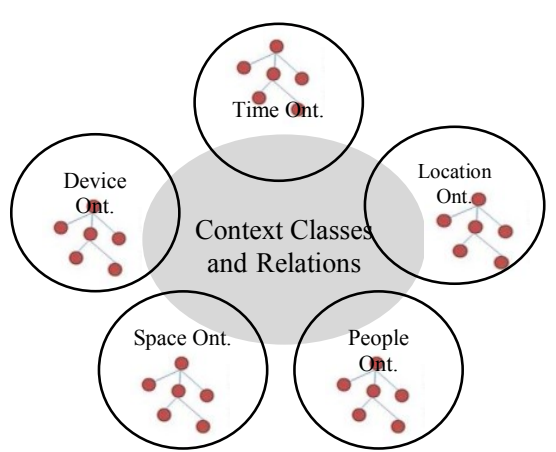

Figure 3 The Context Ontology interrelates all other ontologies

The requirement that the manager might need to call a particular device, creates this loose coupling between the manager and node compulsory.

A vital design task has been the choosing of a development and semantic web framework with which to write the application code. The choice fell on Java and Jena. Jena ${ }^{2}$ provides an RDF and SPARQL API and a rule-based inference engine. This choice was influenced by the fact that Jena, which is a Java-based semantic web library, has robust developer and user backing and has a wider range of APIs and reasoners. In addition, aside from offering an RDF and SPARQL API, Jena boasts high-quality inference support that comprises RDFS and standard rules-based reasoners.

\section{KNOWLEDGEBASE}

On the contrary to PINATA's application code that was deemed necessary to be decentralised across several nodes, the knowledgebase is centralised within the manager application. This approach has two advantages: first and foremost, the node applications remain light and therefore, more favourable to being embedded in devices that are limited when it comes to resources; secondly, when devices are no longer members of PINATA, their data is not lost. The knowledgebase employs a semantic web data model. This option over traditional models arises from three main advantages. Firstly, to understand context the system requires the inference of new implied information from the explicit data sensed by the devices. Secondly, querying becomes simpler and more expressive as the schema language allows data in the knowledgebase to be modelled smartly. Last but not least, the pervasive nature of URIs which identify all resources, makes communication and integration between associated deployments of PINATA easy.

PINATA's data is modelled in RDF and RDFS and SPARQL is being used as a query language. Jena SDB, a component of Jena, is being used as a triple store. It offers scalable storage and querying of RDF datasets through the use

\footnotetext{
${ }^{2} \mathrm{http}: / /$ jena.sourceforge.net/
}

of standard SQL databases. This triple store was chosen so that the Jena framework would be used across the whole application as the SDB is the storage subsystem for Jena; SPARQL is supported by the SDB; and SDB can be installed over reputed DBMSs.

\section{A. ONTOLOGIES}

The scenarios foreseen for the project steered the design of the knowledgebase. The scenarios mainly correspond to circumstances stemming from the sensed data by the pervasive devices. Human beings have knowledge and experience about the ideas that are relevant to a particular context, and how the interrelationships of these notions make them more significant with respect to each other. For this reason, human beings understand context instinctively. A similar line of thought was pursued when designing the ontologies for PINATA. Different components of context were first modelled independently and subsequently, some relations and classes were added that merged the ontologies together to amalgamate the disconnected concepts, as shown in Figure 3. The majority of the ontologies were designed from scratch and include those for devices, persons, pharmaceuticals, space, time, temperature, event and context.

1) The DEVICE Ontology

Several devices are members of PINATA, including Wi-Fi and RFID tags, the Kinect sensor device, handhelds and RFID readers. The device ontology defines the profiles for the different devices, their characteristics and capabilities. Information recorded for the devices includes basic information such as name, brand, manufacturer and semantic web server URIs. The hardware and software capabilities of the devices need to be known as well so that PINATA is able to set up communication using the best available channel; to send the most suitable software client to mobile devices consistent with their platform and library support; and also so as to adjust the output as per the devices' user interface capabilities.

\section{2) The PERSON Ontology}

Different people populate a hospital's environment including doctors, nurses, patients and visitors. These are all stakeholders in PINATA for different reasons. Therefore, a general profile was created for all persons. Furthermore, supplementary information is recorded according to the person's role. For example, for personalisation purposes, device preferences are required for doctors, nurses and visitors. It is important to know the area of specialisation when it comes to medical staff, while when it comes to patients, it is important to record their medical condition and allergies. This ontology is closely related to the hospital field, unlike the device domain ontology.

3) The PHARMACEUTICAL Ontology

A frequent business process within a hospital is that of the administration of pharmaceuticals. PINATA is of great 
advantage here. Other than monitoring the ambient conditions in which the pharmaceuticals are stored, PINATA also alerts medical staff when a patient is prescribed a pharmaceutical that conflicts with their allergies. This ontology describes the ingredients present in the pharmaceutical and any known side effects.

\section{4) The SPACE Ontology}

Devices and personnel are both looked upon as the main resources in the PINATA ambient. Space is another resource which is relevant to correctly identify different contextual situations. For instance, the availability of various kinds and types of rooms is important when scheduling meetings or surgeries. In addition to a description of the space, location is another feature that provides important contextual information on other assets like, the location of people and medical equipment.

\section{5) The TIME Ontology}

A vital context dimension is temporal information. A record of time is frequently essential when a pertinent event occurs. Besides, temporal notions are necessary when scheduling activities.

6) The TEMPERATURE Ontology

A number of PINATA's scenarios revolve around the ability offered by temperature sensors. The ontology caters for the recording of absolute record readings, such as the temperature for refrigerators, as well as for temperature range.

7) The EVENT Ontology

The event ontology caters for events that characterise the day-to-day hospital practices, such as the interaction between a physician and a patient. It caters for scheduled events, like the timely consumption of a pharmaceutical or a scheduled surgery, as well as for unscheduled ones like emergency surgeries and other unannounced situations such as a patient falling and requiring treatment. Apart from these, the ontology also caters for semi-scheduled events which are events occurring in a particular time frame but the specific instance when they occur cannot be foreseen, like for example, a visitor visiting a relative.

8) The CONTEXT Ontology

Up to now, the ontologies catered for different resources and context dimensions with no reference to how they will relate to each other. In real life, however, these separate models are hardly ever thought of in isolation. Instead, context information is thought of as the compilation of distinct but associated information pertaining to a specific situation. The context ontology brings in some further relations that connect the ontologies described so far.

\section{B. CONTEXT}

The additional relations that connect the rest of the ontologies include those between tangible resources, location and time; pharmaceuticals, temperature and patients; events, location, time and people. Initially, the idea that was considered was that tangible resources have a location at different times. Then, pharmaceuticals were related to the notions of temperature and patients. A drug normally has a recommended temperature or temperature range in which it is to be stored. In addition, a relation between patients and pharmaceuticals was also catered for. Lastly, just as there is the need to store information regarding location and time of tangible resources, the same applies for other abstract situations like patient's appointments. Besides location and time, events also have one or more persons related to them.

\section{EVALUATION}

The modules related to the tracking of patients and other resources have been coded and tested using dummy values. The results have been very encouraging to say the least. The evaluation is being based on the scenarios developed for the design of the system, and the success of the system is being based on whether it is managing the scenarios as planned.

The scenarios that we used include: (i) a fire in the hospital; (ii) patients or visitors stepping into unauthorised zones; (iii) persons exiting the building in an unauthorised manner; (iv) patient's data being sent to staff's handhelds when these enter the patient's room; (v) patients being in the vicinity of incompatible medicine; (vi) wrong ambient conditions for refrigerators and products; (vii) equipment being lost in the hospital; (viii) request for statistical information like which equipment or products were used on which patients; and (ix) request for the location of PINATA's devices. These scenarios were developed after discussion with the hospital ${ }^{3}$ staff which is also contributing to PINATA. The following results have been deemed successful:

- The system was able to report on the presence and location for people as shown in Table 1.

- It was able to advise staff and visitors as to where the nearest exit is in case of an emergency as shown in Table 2. Presently, all output is being viewed on a desktop computer.

- The system was able to detect and differentiate the presence of patients and visitors in areas deemed as unauthorised and to identify and alert the nearest member of staff as can be seen in Tables 3 and 4 .

- $\quad$ PINATA was also able to successfully detect and identify patients exiting the building in an unauthorised manner and to identify and alert their carer in order to take the appropriate action as shown in Table 4.

- The system is also able to monitor temperature tags attached to a refrigerator or to a product, such as

\footnotetext{
${ }^{3}$ St James' Hospital Malta
} 
blood and to alert hospital staff when the ambient conditions changed or were not correct as can be seen in Table 5.

- $\quad$ PINATA was able to successfully locate and keep track of the various types of equipment within the hospital.

- The system was also able to duly report on data collected, such as which equipment and products were used on which patient, the patients' average waiting time to be seen by a consultant and how much patients a consultant visited during a particular time frame.

Other scenarios which we explored involve the use of the Kinect cameras. These cameras were used in order to monitor patients and notify staff when necessary. The considered scenarios include (i) a patient falling in his room; (ii) monitoring of whether the patient's gait is improving and whether it is signaling potential problems; (iii) a patient lying on the bed for longer than $\mathrm{s} /$ he normally sleeps. The visibility of the camera is going to be semantically tagged, so that it would be possible to make statements indicating what a patient was doing at a particular time.

Further, more thorough evaluation is planned in collaboration with St James Hospital ${ }^{4}$ so that the behavior and the robustness of the system are monitored during the normal activities within the hospital. .

\section{CONCLUSION}

By offering context-driven information on handhelds, PINATA seeks to help the nursing and doctoral staff to perform their duties and to give high quality healthcare. So as to attain this objective, the most recent developments in the applicable technological building blocks have been explored, including: devices (such as RTLS, Kinects, handhelds and mobile phones), wireless networks (Wi-Fi and Bluetooth), context and domain ontologies; and AmI architectures. PINATA is an ambitious project that requires the application of tools and techniques from multidisciplinary areas. It is at the forefront of new AmI projects because of the combination of the latest research developments and the utilisation of artefacts originating from primary research.

PINATA's architecture consists of thin decentralised nodes and a centralised manager acting as an orchestrator for the nodes' functionality. The PINATA knowledgebase is an $\mathrm{RDF} / \mathrm{S}$ data model with several newly designed and developed ontologies representing conceptualisations that
TABLE 1 LOCATING PEOPLE IN CASE OF EMERGENCY

\begin{tabular}{|l|l|}
\hline \multicolumn{1}{|c|}{ Person } & \multicolumn{1}{c|}{ isLocatedAt } \\
\hline $411277(\mathrm{M})$ & operatingTheatre \\
$404572(\mathrm{M})$ & recoveryroom \\
11111113 & recoveryroom \\
$123554(\mathrm{M})$ & exit1 \\
$966774(\mathrm{M})$ & operatingTheatre \\
11111111 & storeroom \\
$547857(\mathrm{M})$ & reception \\
$633475(\mathrm{M})$ & operatingTheatre \\
\hline
\end{tabular}

TABLE 2 DIRECTING PEOPLE TO THE NEAREST EXIT

\begin{tabular}{|l|l|}
\hline \multicolumn{1}{|c|}{ People } & Exit \\
\hline $411277(\mathrm{M})$ & Exit1 \\
633475 & Exit2 \\
$404572(\mathrm{M})$ & Exit1 \\
11111113 & Exit1 \\
$123554(\mathrm{M})$ & Exit1 \\
$966774(\mathrm{M})$ & Exit1 \\
11111111 & Exit1 \\
$547857(\mathrm{M})$ & Exit2 \\
$633475(\mathrm{M})$ & Exit1 \\
$111572(\mathrm{M})$ & Exit 1 \\
$256853(\mathrm{M})$ & Exit2 \\
11111112 & Exit1 \\
633474 & Exit1 \\
$215553(\mathrm{M})$ & Exit1 \\
\hline
\end{tabular}

TABLE 3 DETECTING PEOPLE IN UNAUTHORISED ZONES

\begin{tabular}{|c|c|}
\hline Person & Room \\
\hline $404572(\mathrm{M})$ & recoveryroom \\
11111113 & recoveryroom \\
$123554(\mathrm{M})$ & exit1 \\
11111111 & storeroom \\
$547857(\mathrm{M})$ & reception \\
$633475(\mathrm{M})$ & operatingTheatre \\
$256853(\mathrm{M})$ & exit2 \\
11111112 & operatingTheatre \\
$215553(\mathrm{M})$ & storeroom \\
\hline
\end{tabular}

TABLE 4 ALERTING NEARBY STAFF AND/OR CARERS

\begin{tabular}{|c|}
\hline Near_nurse \\
\hline \hline 633475 \\
$300178(\mathrm{M})$ \\
\hline \hline \\
\hline \hline Carer \\
\hline \hline 300178(M) \\
\hline
\end{tabular}

TABLE 5 DETECTING WRONG TEMPERATURES

\begin{tabular}{|c|c|}
\hline Fridge & Alert \\
\hline Sensorblood01 & High_temperature_alert \\
Sensorfridge01 & Low_temperature_alert \\
\hline
\end{tabular}


humans have and that are significant to the project's requirements.

The success of the preliminary evaluation was based on whether the system is coping with the envisaged scenarios as planned. As discussed in the evaluation section, the initial results have been encouraging and showed that the system was able to cater for most of the scenarios planned. Further evaluation however is in the pipe line, this time with real time values obtained through the devices. We also plan to deploy the system in a ward within the hospital to be able to fully realise the capabilities of PINATA in a real hospital setting. It is envisaged that with further improvement of the visual tracking component and better integration of the handhelds, the system will cater better for all the envisaged scenarios.

\section{ACKNOWLEDGEMENTS}

This work was done within the PINATA project which is financed by the Malta Council for Science and Technology ${ }^{5}$ and done in association with St. James Hospital Malta.

\section{REFERENCES}

1. IST Advisory Group (ISTAG): Ambient Intelligence: from Vision to Reality. Consolidated Draft Report, ISTAG (2005)

2. Kummer, Tyge-F., Bick, Markus, Gururajan, Raj: Acceptance Problems Of Ambient Intelligence And Mobile Technologies In Hospitals In India And Germany. In : 17th European Conference on Information Systems, Verona (2009)

3. Andersen, J., Bardram, J.E.: BLIG: A New Approach for Sensor Identification, Grouping, and Authorisation in Body Sensor Networks. In : Proceedings 4th International Workshop on Wearable and Implantable Body Sensor Networks (BSN 2007), pp.223-229 (2007)

4. Dingli, Alexiei, Abela, Charlie: A Pervasive Assistant For Nursing and Doctoral Staff (PINATA). In : European Semantic Web Conference (2008)

5. Rudolph, Larry: Project Oxygen: Pervasive, Human-Centric Computing An Initial Experience. 13th International Conference in Advanced Information 2068, 1-12 (2001)

6. Janse, Maddy, Vink, Peter, Georgantas, Nikolaos: Amigo Architecture: Service Oriented Architecture for Intelligent Future In-Home Networks. In : 5th Working IEEE/IFIP Conference on Software Architecture (2005)

7. Sarnovsky, Martin, Kostelnik, Peter, Burka, Peter, Jan, Hreno, Lackova, Dasa: First Demonstrator of HYDRA Middleware Architecture for Building Automation. Znalosti (2008)

8. Ashruf, Colin: Ambient Intelligence, Coming To You. Password - Philips Research Technology Magazine(23), 6-11 (May 2005)

9. Rodríguez, Marcela, Favela, Jesus, Preciado, Alfredo, Vizcaíno, Aurora: Agent-based ambient intelligence for healthcare. AI Communications

\footnotetext{
${ }^{5} \mathrm{http}: / /$ www.mcst.gov.mt
}

18(3), 201-216 (2005)

10. Huang, G.T.: Monitoring Mom, As population matures, so do assistedliving technologies. Technology Review (July/August 2003)

11. Hartog, F., Schmidt, J.R, Vries, A.: On the potential of personal networks for hospitals. International Journal of Medical Informatics 75(9), 658-663 (2006)

12. Little, Linda, Briggs, Pam: Using AmI systems for exchanging health information: Considering trust and privacy issues., PACT Lab, Northumbria University, UK (2007)

13. P, Rubel, J, Fayn, L, Simon-Chautemps, H, Atoui, M, Ohlsson, D, Telisson, S, Adami, S, Arod, MC, Forlini, C, Malossi, J, Placide, GL, Ziliani, D, Assanelli, P., Chevalier: New paradigms in telemedicine: ambient intelligence, wearable, pervasive and personalized. Studies in Health, Technology and Informatics 108, 123-32 (2004)

14. Favela, Jesús, Martínez, Ana, Rodríguez, Marcela, González, Víctor: Ambient Computing Research for Healthcare: Challenges, Opportunities and Experiences. Computación y Sistemas 12(1), 109-127 (julioseptiembre 2008)

15. Spanakis, M., Lelis, P., Chiarugi, F., Chronaki, C., Tsiknakis, M.: R\&D Challenges In Developing An Ambient Intelligence E-Health Platform., Foundation for Research and Technology - Hellas, Heraklion, Crete, Greece (2007)

\section{APPENDIX}

TABLE 6 COMPARISON OF CITED WORK IN COMPARISON WITH PINATA

\begin{tabular}{|c|c|c|c|c|c|c|c|c|c|c|}
\hline 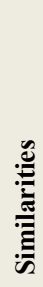 & 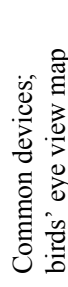 & 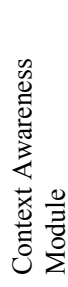 & 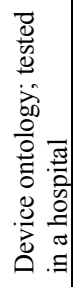 & 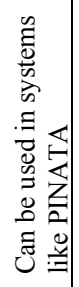 & 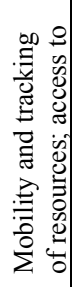 & 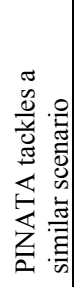 & 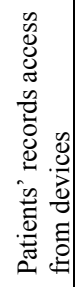 & 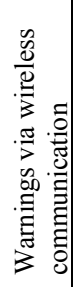 & 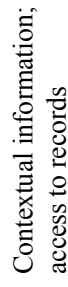 & 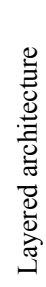 \\
\hline 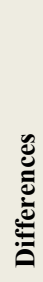 & 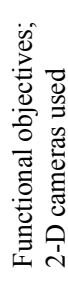 & 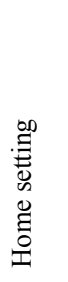 & 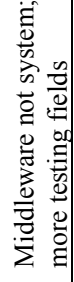 & 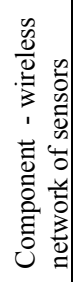 & 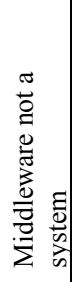 & 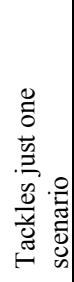 & 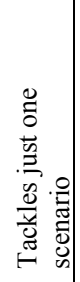 & 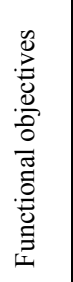 & 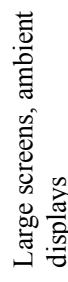 & 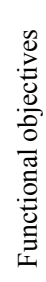 \\
\hline 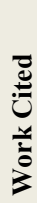 & 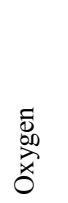 & $\frac{\stackrel{\circ}{E}}{\mathbb{E}}$ & 吾 & ర్ల & $\begin{array}{l}\mathbb{S} \\
\text { 岕 } \\
\underset{\sim}{4}\end{array}$ & 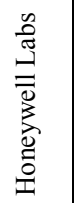 & $\begin{array}{l}\bar{\pi} \\
\tilde{0} \\
00 \\
0 \\
0 \\
\mathbb{J} \\
\mathbb{J}\end{array}$ & $\sum_{\substack{\mid=1 \\
2}}$ & 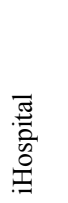 & $\frac{\pi}{\mathbb{Z}}$ \\
\hline
\end{tabular}

